\title{
Effects of preservation techniques on in vivo expression of adhesion molecules by aortic valve allografts
}

\begin{abstract}
Methods of sterilization and preservation of aortic valve allografts influence graft longevity. The effect of storage techniques on valve durability may be mediated by alterations in the immunologic properties of the allograft, which are reflected by expression of leukocyte adhesion molecules. Rat aortic valve grafts were transplanted in the fresh state, after cryopreservation $\left(-196^{\circ} \mathrm{C}\right.$, or after storage at $4^{\circ} \mathrm{C}$ for 1 to 21 days. Syngeneic and strongly allogeneic valves were transplanted for 4 hours to 21 days and were retrieved for immunohistochemical staining for expression of leukocyte adhesion molecules. Unimplanted valves and transplanted syngeneic valves, regardless of storage methods, exhibited little or no expression of leukocyte adhesion molecules. Fresh allogeneic valves expressed all molecules, indicating up-regulation, at all time intervals studied. Cryopreserved allogeneic valves demonstrated no leukocyte adhesion molecules at 4 hours or 2 days and weak reactivity at 10 and 21 days. Allogeneic valves stored at $4^{\circ} \mathrm{C}$, regardless of the duration of storage, demonstrated weak expression of all molecules at 10 days and strong expression at 21 days. Expression of leukocyte adhesion molecules requires an allogeneic environment and may precede immune-mediated injury. Reduced expression of leukocyte adhesion molecules resulting from storage may predict a diminished immunologic response. Cryopreservation $\left(-196^{\circ} \mathrm{C}\right)$ causes the greatest delay and diminution of expression of leukocyte adhesion molecules. (J ThORAC CARDIOvasC SURG 1994;107:717-23)
\end{abstract}

Michael S. Mulligan, MD, Thomas T. Tsai, James M. Kneebone, MS, Peter A. Ward, MD, and Flavian M. Lupinetti, MD, Ann Arbor, Mich.

$\mathrm{H}$ uman valve allografts have been successfully used to treat a number of cardiac diseases. Improved methods of valve treatment and storage have improved both the availability and the longevity of these tissues. In some centers, allograft valves are stored at $4^{\circ} \mathrm{C}$, and satisfactory clinical results have been reliably achieved. ${ }^{1}$ More recently, cryopreservation of allograft valves has succeeded in improving the availability of these scarce resources. Cryopreservation may result in diminished endothelial

\footnotetext{
From the Department of Pathology and the Department of Surgery, Section of Thoracic Surgery, The University of Michigan School of Medicine, Ann Arbor, Mich.

Supported by grant R01 HL 42426 from the National Heart, Lung, and Blood Institute.

Received for publication Jan. 21, 1993.

Accepted for publication June 29, 1993.

Address for reprints: Flavian M. Lupinetti, MD, Children's Hospital and Medical Center, 4800 Sand Point Way, Seattle, WA 98105.

Copyright $\odot 1994$ by Mosby-Year Book, Inc.

$0022-5223 / 94 \$ 3.00+0 \quad 12 / 1 / 50465$
}

cellularity and in a reduction in the expression of class I surface antigens. These phenomena may be related, because endothelial cells constitute the major source of antigen presentation. ${ }^{2}$ However, endothelial cells play an important role in preventing thrombus formation, inhibiting calcification, and providing nutrition to the fibroblasts of the allograft. ${ }^{3}$ Thus whether the loss of endothelial cells has a positive, negative, or neutral effect on longterm allograft structure and function remains unknown. In this regard, O'Brien and colleagues ${ }^{4}$ have observed superior long-term performance with cryopreserved allograft valves, surpassing even those results obtained in their prior experience using fresh allografts.

Expression of the leukocyte adhesion molecules by vascular beds predicts inflammatory response. Endothelium-leukocyte adhesion molecule-1 (ELAM-1), for example, is required in neutrophil-mediated lung injury in rats. ${ }^{5}$ Intercellular adhesion molecule-1 (ICAM-1) is expressed on canine cardiac monocytes ${ }^{6}$ and is a mediator of cardiac allograft rejection in cynomolgus monkeys. ${ }^{7}$ 
In both acute cardiac allograft rejection and chronic kidney allograft rejection in rats there is up-regulation of ICAM-1 and increased ICAM-1 specific binding of lymphocytes. ${ }^{8,9}$ Expression of vascular cell adhesion molecule-1 (VCAM-1) is detected in murine cardiac allografts beginning 3 days after transplantation or 3 to 4 days before rejection is complete. ${ }^{10,11}$

Previous studies investigating these three important molecules examined fresh tissues. Whether tissues stored for prolonged periods at $4^{\circ} \mathrm{C}$ or those subjected to cryopreservation express these molecules is unknown. The present studies were designed to examine the effects of preservation methods and immunologic differences on adhesion molecule expression by in vivo aortic valve grafts. Because adhesion molecule expression effects lymphocyte migration and accumulation, ${ }^{8}$ the modulation of adhesion molecule expression according to the selected method of graft preservation may affect the immune response. This may in turn affect long-term survival and function of valve allografts.

\section{Materials and methods}

Valve harvest, sterilization, and preservation. Male rats, 125 to $175 \mathrm{gm}$ body weight, of the Lewis and Brown-Norway strains (strains that are strongly histoincompatible at both the RT1 and the non-RT1 loci) were subjected to general pentobarbital anesthesia. A median sternotomy was performed. The aortic valve with a short portion of ascending aorta was excised and rinsed in chilled, heparinized saline. Some valves were transplanted immediately. Other valves were placed in chilled saline, placed on ice, and transported to CryoLife, Inc. (Marietta, Ga.), for cryopreservation. At the time of implantation, the valves were thawed by immersion in $37^{\circ} \mathrm{C}$ saline and rinsed thoroughly before use. The remaining valves were placed in $4^{\circ}$ C RPMI 1640 tissue medium containing $10 \%$ fetal calf serum and the following antibiotics: gentamicin $(160 \mathrm{mg} / \mathrm{L})$, piperacillin $(1000 \mathrm{mg} / \mathrm{L})$, oxacillin $(50 \mathrm{mg} / \mathrm{L})$, metronidazole $(200$ $\mathrm{mg} / \mathrm{L})$, and amphotericin $\mathrm{B}(100 \mathrm{mg} / \mathrm{L})$. This combinations of antibiotics was selected on the basis of two previous studies, one from this laboratory, which showed that this solution resulted in endothelial viability without bacterial growth after as much as 3 weeks of storage at $4^{\circ} \mathrm{C},{ }^{12}$ and one from Strickett, BarrattBoyes, and MacCulloch, ${ }^{13}$ which characterized the tissue toxicity of other antibiotics previously used for allograft sterilization. ${ }^{13}$ After 24 hours, the tissues were transferred to RPMI 1640 culture medium with $10 \%$ fetal calf serum without antibiotics at $4^{\circ} \mathrm{C}$. The tissue was maintained in this solution for $1,3,7,10,14$, or 21 days. At the time of implantation, the valves were rinsed in $0.9 \%$ saline solution before use.

Operative preparation. Heterotopic transplantation of aortic valve allografts was performed as described originally by Yankah and colleagues. ${ }^{14}$ Male Brown-Norway rats, 175-250 gm body weight, underwent general anesthesia with sodium pentobarbital, $60 \mathrm{mg} / \mathrm{kg}$ body weight, administered by intraperitoneal injection. Rats underwent laparotomy and dissection of the abdominal aorta under a $14 \times$ operating microscope. The recipient aorta was occluded proximally and distally and divided. An end-to-end interposition of the allograft was performed with 8-0 polypropylene suture. The anterior leaflet of the aortic valve was incorporated into the suture line to prevent valve competence, which may predispose to thrombosis. The abdomen was closed and the animal allowed to recover. Animals received humane care in compliance with the "Guide for the Care and Use of Laboratory Animals" published by the National Institutes of Health (NIH Publication No. 86-23, revised 1985).

Valve retrieval and preparation. The valves remained in place for 4 hours, 2 days, 10 days, or 21 days. After the designated interval the animals were again subjected to general anesthesia, the grafts were retrieved, and the animals were killed. The retrieved grafts were rinsed in saline and washed free of salts with distilled water. They were then embedded in Tissue-Tek O.C.T. (Miles Inc., Elkhart, Ind.) and frozen in liquid nitrogen. Sections were cut $8 \mu \mathrm{m}$ thick and mounted on poly-Llysine-coated slides for immunoperoxidase staining. Two or three valves of each strain, with each method of preservation, for each duration of implantation, were studied. Fifty sections of each specimen were stained and graded for evaluation in a blinded fashion.

Monoclonal antibodies. The antibodies used for immunoperoxidase staining were all monoclonal immunoglobulin $\mathrm{G}$, and were directed against ELAM-1 (CL-3), ICAM-1 (1A29) or VCAM-1 (CL-348). CL-3 (a gift from C. W. Smith, Baylor College of Medicine, Houston, Tex.) was prepared by immunization of BALB/C mice with interleukin-1-stimulated endothelial cells from the human umbilical vein. After hybridoma formation and subcloning the antibody was found to be crossreactive with interleukin-1-stimulated rat endothelial cells but not leukocyte populations. ${ }^{5}$ 1A29 (a gift from M. Miyasaka, Tokyo Metropolitan Institute of Medical Science, Tokyo, Japan) was raised specifically against the rat ICAM-1 homolog as previously described. ${ }^{15}, 16$ CL-348 (a gift from R. Lobb, Biogen Corporation, Cambridge, Mass.) was raised against the human antigen and was found to be cross-reactive in the rat. ${ }^{17-19}$

Frozen sections were mounted on poly-L-lysine-coated glass slides, fixed with methanol containing $0.3 \%$ hydrogen peroxide at room temperature for 30 minutes, and washed with phosphate-buffered saline. They were then incubated with CL-3, CL-348, and 1A29 for 45 minutes. For control studies, other sections were similarly incubated with a nonspecific immunoglobulin G (MOPC 21, mouse myeloma). The slides were washed with phosphate-buffered saline and stained for bound monoclonal antibodies with the Vectastain biotin/avidin-peroxidase system for mouse immunoglobulin $\mathrm{G}$ (Vector Laboratories Inc., Burlingame, Calif.). After hematoxylin counterstaining, sections were coated with Aquamount (Lerner Laboratories, Pittsburgh, Pa.) and examined by light microscopy for the presence of reaction products of peroxidase.

\section{Results}

The results of the immunohistochemical staining studies are summarized in Tables I to III. Unimplanted valves, regardless of storage methods, were not reactive for ICAM-1, VCAM-1, or ELAM-1 (Fig. 1). Syngeneic valves that were transplanted in the fresh state did not stain for the adhesion molecules at the 4-hour or 2-day 


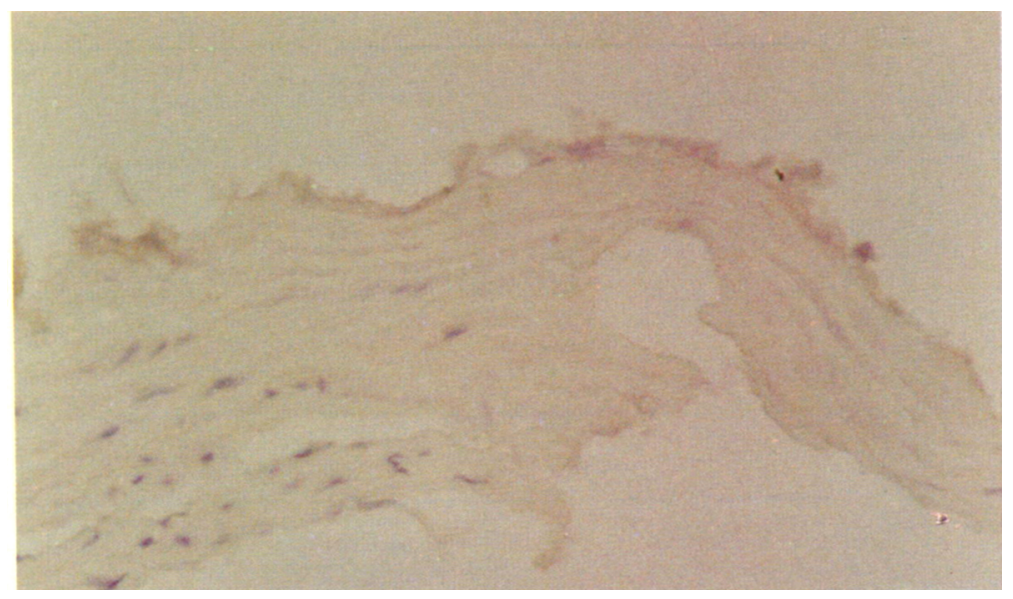

Fig. 1. Allogeneic valve implanted after cryopreservation and retrieved after 2 days, stained for ELAM-1, and counterstained with hematoxylin. Absence of reddish-brown coloration indicates absence of ELAM-1. $(\times 220$.)

Table I. Expression of adhesion molecules by untransplanted aortic valve grafts

\begin{tabular}{cccccccc}
\hline & \multicolumn{7}{c}{ Storage method } \\
\cline { 2 - 8 } & Fresh & Cryopreserved & $4^{\circ} \mathrm{C} \times 1$ day & $4^{\circ} \mathrm{C} \times 3$ days & $4^{\circ} \mathrm{C} \times 7$ days & $4^{\circ} \mathrm{C} \times 14$ days & $4^{\circ} \mathrm{C} \times 21$ days \\
\hline ELAM-1 & - & - & - & - & - & - & - \\
ICAM-1 & - & - & - & - & - & - & - \\
VCAM-1 & - & - & - & - & - & - & - \\
\hline
\end{tabular}

+ , Positive staining for indicated adhesion molecule; - , absence of staining for molecule; \pm , weak or minimal staining for molecule.

Table II. Expression of adhesion molecules by transplanted syngeneic aortic valve grafts

\begin{tabular}{|c|c|c|c|c|c|c|c|}
\hline \multirow{2}{*}{$\begin{array}{c}\text { Duration } \\
\text { implanted }\end{array}$} & \multicolumn{7}{|c|}{ Storage method } \\
\hline & Fresh & Cryopreserved & $4^{\circ} \mathrm{C} \times 1$ day & $4^{\circ} \mathrm{C} \times 3$ days & $4^{\circ} \mathrm{C} \times 7$ days & $4^{\circ} \mathrm{C} \times 14$ days & $4^{\circ} \mathrm{C} \times 21$ days \\
\hline \multicolumn{8}{|l|}{4 Hours } \\
\hline ELAM-1 & - & - & - & - & - & - & - \\
\hline ICAM-1 & - & - & - & - & - & - & - \\
\hline VCAM-1 & - & - & - & - & - & - & - \\
\hline \multicolumn{8}{|l|}{2 Days } \\
\hline ELAM-1 & - & - & - & - & - & - & - \\
\hline ICAM-1 & - & - & - & - & - & - & - \\
\hline VCAM-1 & - & - & - & - & - & - & - \\
\hline \multicolumn{8}{|l|}{10 Days } \\
\hline ELAM-1 & - & - & - & - & - & - & - \\
\hline ICAM-1 & \pm & - & - & - & - & - & - \\
\hline VCAM-1 & - & - & - & - & - & - & - \\
\hline \multicolumn{8}{|l|}{21 Days } \\
\hline ELAM-1 & \pm & - & - & - & - & - & - \\
\hline ICAM-I & \pm & - & - & - & - & - & - \\
\hline VCAM-1 & \pm & - & - & - & - & - & - \\
\hline
\end{tabular}

+ , Positive staining for indicated adhesion molecule; - , absence of staining for molecule; \pm , weak or minimal staining for molecule.

intervals. At 10 days there was only minimal reactivity for ICAM-1 and no reactivity for ELAM-1 or VCAM-1. By 21 days the fresh syngeneic valves demonstrated minimal reactivity for all three adhesion molecules. Cryopreserved syngeneic valves and syngeneic valves stored at $4^{\circ} \mathrm{C}$ for any duration showed no reactivity for ICAM-1, ELAM1, or VCAM-1 at any of the time points examined.

Allogeneic valves that were transplanted in a fresh state 
Table III. Expression of adhesion molecules by transplanted allogeneic aortic valve grafts

\begin{tabular}{|c|c|c|c|c|c|c|c|}
\hline \multirow{2}{*}{$\begin{array}{c}\text { Duration } \\
\text { implanted }\end{array}$} & \multicolumn{7}{|c|}{ Storage method } \\
\hline & Fresh & Cryopreserved & $4^{\circ} \mathrm{C} \times 1$ day & $4^{\circ} \mathrm{C} \times 3$ days & $4^{\circ} \mathrm{C} \times 7$ days & $4^{\circ} \mathrm{C} \times 14$ days & $4^{\circ} \mathrm{C} \times 21$ days \\
\hline \multicolumn{8}{|l|}{4 Hours } \\
\hline ELAM-1 & + & - & - & - & - & - & - \\
\hline ICAM-1 & + & - & - & - & - & - & - \\
\hline VCAM-1 & + & - & - & - & - & - & - \\
\hline \multicolumn{8}{|l|}{2 Days } \\
\hline ELAM-1 & + & - & - & - & - & - & - \\
\hline ICAM-1 & + & - & - & - & - & - & - \\
\hline VCAM-1 & + & - & - & - & - & - & - \\
\hline \multicolumn{8}{|l|}{10 Days } \\
\hline ELAM-1 & + & \pm & \pm & \pm & \pm & \pm & \pm \\
\hline ICAM-1 & + & + & \pm & \pm & \pm & \pm & \pm \\
\hline VCAM-1 & + & - & \pm & \pm & \pm & \pm & \pm \\
\hline \multicolumn{8}{|l|}{21 Days } \\
\hline ELAM-1 & + & \pm & + & + & + & + & + \\
\hline ICAM-1 & + & + & + & + & + & + & + \\
\hline VCAM-1 & + & \pm & + & + & + & + & + \\
\hline
\end{tabular}

+ , Positive staining for indicated adhesion molecule; - , absence of staining for molecule; \pm , weak or minimal staining for molecule.

showed strong reactivity at all time points examined for ELAM-1, ICAM-1, and VCAM-1 (Fig. 2). When allogeneic donor valves underwent cryopreservation before implantation, there was delayed and diminished expression of all three adhesion molecules. In contrast to the freshly transplanted allografts, cryopreserved allografts showed no reactivity at 4 hours or 2 days for any of the adhesion molecules. Staining for ELAM-1 did not become positive until 10 days after transplantation and even then it was relatively weak. By 21 days, the staining for ELAM-1 had decreased considerably. Moderate staining for ICAM-1 was evident at 10 days and persisted at 21 days. Staining for VCAM-1 was not evidenced until 21 days after transplantation and even then was minimal. The allogeneic valves stored at $4^{\circ} \mathrm{C}$ showed a uniform response for all durations of storage. No adhesion molecules were expressed at 4 hours or 2 days, all were expressed weakly at 10 days, and all were expressed strongly at 21 days. Inflammatory infiltrates were observed in grafts in proportion to the intensity of adhesion molecule expression (Fig. 3).

\section{Discussion}

The immunologic stimulus caused by an allogeneic mismatch appears capable of inducing adhesion molecule expression by heterotopically transplanted aortic valves. The present studies further indicate that subjecting donor valves to a period of cryopreservation delays and suppresses the regulation of the endothelial adhesion molecule. This could result from loss of endothelial cells from the donor grafts after preservation. Previous investigations from this laboratory have shown that cryopreserved human arteries and valves retain identifiable endothelial cells in only $16 \%$ of specimens examined. ${ }^{20}$ Loss of donor endothelium may increase the possibility that host endothelium ultimately resurfaces the donor valve. Other models have shown host endothelial repopulation of allograft vasculature. ${ }^{21,22}$ Because endothelial antigens present the primary immunologic stimulus, such an effect of cryopreservation may delay adhesion molecule expression (while endothelial surfaces are regenerated). Furthermore, reendothelialization by host endothelial cells could render the valves less immunogenic, and adhesion molecule expressions would presumably be diminished. This proposed sequence of events would agree precisely with the results seen in our studies after cryopreservation.

Regardless of the mechanism, the attenuation of adhesion molecule expression by the application of preservation techniques may diminish immune-mediated destruction of transplanted valves. Endothelial cells express adhesion molecules when stimulated by cytokines, thereby stimulating leukocyte adherence (Fig. 4). This observation is supported by recent findings wherein monoclonal antibodies to various adhesion molecules have been used to protect against acute inflammatory injury. ELAM-1 is required for the development of lung injury and the accumulation by neutrophils in lungs after the deposition of immune complexes. ${ }^{5}$ VLA-4 is the ligand for VCAM-1, and infusion of an anti-VLA-4 inhibits adherence of rat T-cells to VCAM-1 on stimulated endothelial cells. Anti-VLA-4 also selectively inhibits migration of lymphocytes enriched in T memory cells into sites of cutaneous and joint inflammation. ${ }^{23} \mathrm{CD} 18$ is the common $\beta$-subunit of the $\mathrm{B}_{2}$ integrin adhesion mol- 




Fig. 2. Allogeneic valve implanted fresh and retrieved after 2 days, stained for ELAM-1, and counterstained with hematoxylin. Reddish-brown coloration indicates presence of ELAM-1. (×220.)

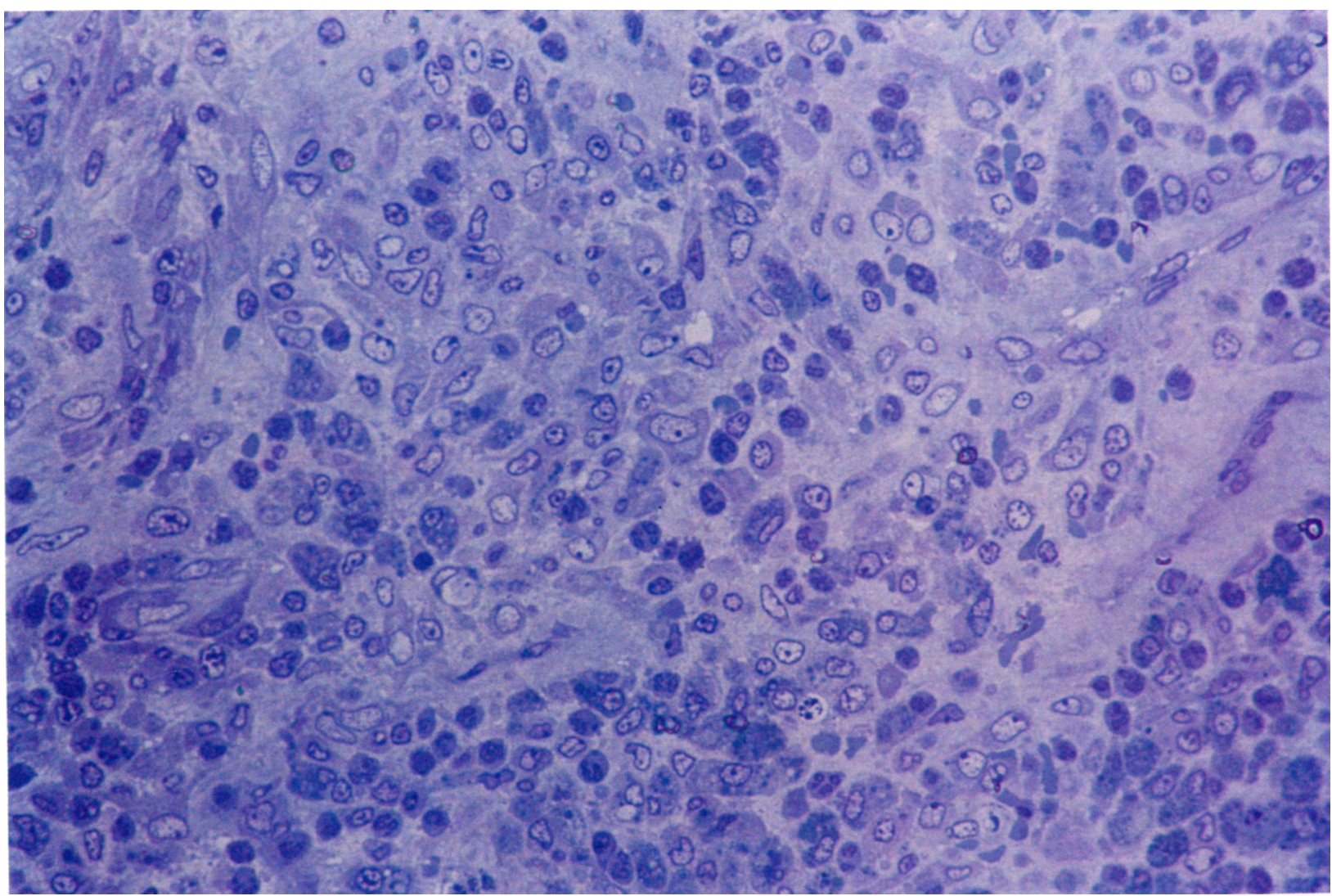

Fig. 3. Fresh allogeneic valve implanted for 10 days. An acute inflammatory infiltrate is demonstrated. $(\times 190$.)

ecules. Two of the $\mathrm{B}_{2}$ integrins, LFA-1 and MAC-1, are recognized by ICAM-1. Murine monoclonal antibodies to $\mathrm{CD} 18$ and ICAM-1 in various animal models have also demonstrated that the CD18 integrins and ICAM-1 are required for leukocyte accumulations at inflammatory sites. ${ }^{24,}{ }^{25}$ Anti-ICAM-1 has been used to prolong renal and cardiac allograft survival in monkeys. ${ }^{7}$

The importance of immunologic differences between 


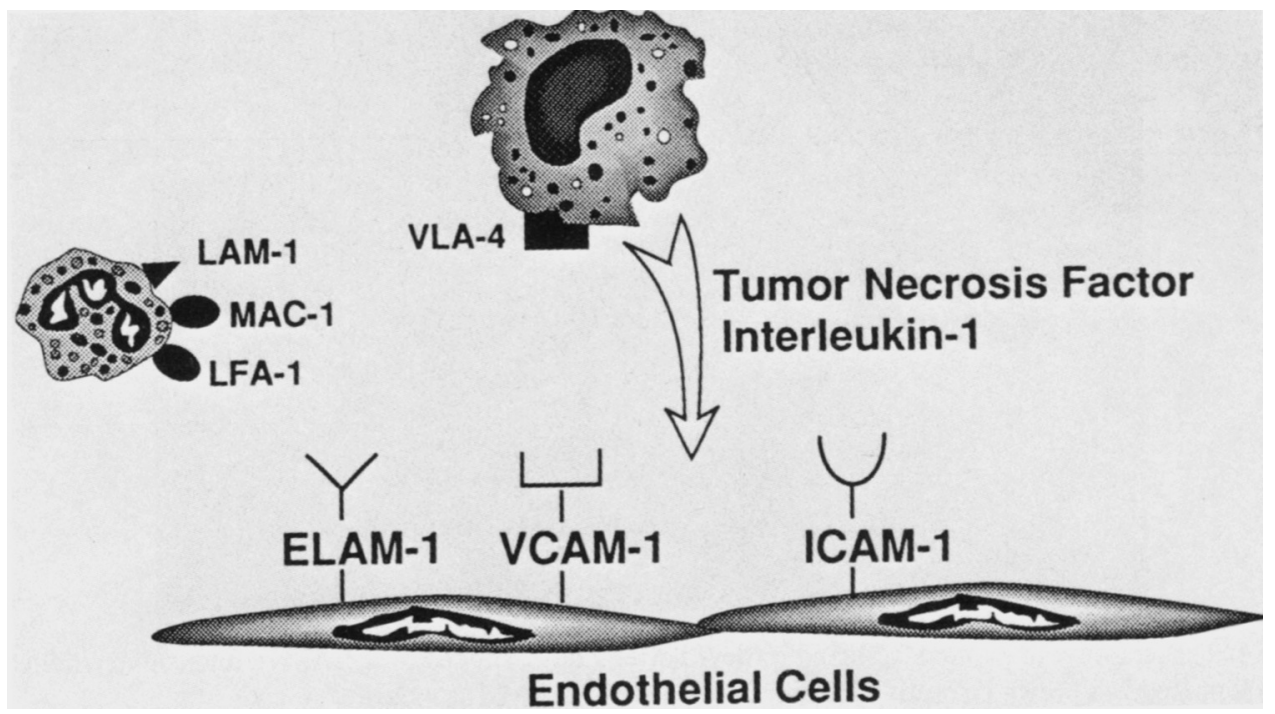

Fig. 4. Vascular endothelial cells when stimulated by tumor necrosis factor, interleukin-1, or other cytokines express the adhesion molecules ELAM-1, VCAM-1, and ICAM-1. The ligands for these molecules include LAM-1, MAC1 , and LFA-1, which are found on neutrophils, and VLA-4, which is found on monocytes.

donor and recipient on the structure and function of aortic valve allografts remains uncertain. Current clinical practices regarding allograft use place little emphasis on tissue typing of donors and recipients, immunosuppression of allograft recipients, or avoidance of allografts in previous allograft recipients. Studies in patients with ABO blood group-compatible valves compared with those with incompatible valves have demonstrated no differences in allograft longevity. Nevertheless, it is the opinion of many practitioners that $\mathrm{ABO}$ matching is preferred if circumstances permit. ${ }^{26}$

Evidence that allograft immunogenicity may affect graft function has included isolated reports of accelerated calcification in recipients of multiple allografts. This evidence is supported by experimental studies from this and other laboratories that observed a positive correlation between immunologic differences and allograft calcification..$^{27,28}$ If immunologic differences are important in determining the pathologic fate of allograft valves, it is possible that methods of harvest, storage, and preservation should be tailored to minimize such differences. Cryopreservation or storage at $4^{\circ} \mathrm{C}$ may both be acceptable methods for doing so. The outstanding results that have been reported with cryopreserved valve allografts may in part be a reflection of more profound immunologic alterations.

In summary, ELAM-1 has been demonstrated for the first time to be up-regulated in transplanted tissues. VCAM-1 and ICAM-1, which have previously been observed in whole organ transplants, are observed in allograft valves as well. The expression of each of these adhesion molecules by allograft valves is attenuated by clinically used methods of graft storage. This attenuation of inflammatory mediators may in turn blunt the host response to the valves, perhaps improving graft longevity.

\section{REFER E N C ES}

1. Matsuki O, Robles A, Gibbs S, Bodnar E, Ross DN. Long-term performance of 555 aortic homografts in the aortic position. Ann Thorac Surg 1988;46:187-91.

2. Pober JS, Collins T, Gimbrone MA Jr, Libby P, Reiss CS. Inducible expression of class II major histocompatibility complex antigens and the immunogenicity of vascular endothelium. Transplantation 1986;41:141-6.

3. Jaffe EA. Cell biology of endothelial cells. Hum Pathol 1984;18:234-9.

4. O'Brien MF, McGiffin DC, Stafford EG, et al. Allograft aortic valve replacement: long-term comparative clinical analysis of the viable cryopreserved and antibiotic $4^{\circ} \mathrm{C}$ stored valves. J Cardiac Surg 1991;6(Suppl):534-43.

5. Mulligan MS, Varani J, Dame MK, et al. Role of endothelial-leukocyte adhesion molecule 1 (ELAM-1) in neutrophil mediated lung injury in rats. J Clin Invest 1991; 88:1396-1406.

6. Smith CW, Entman ML, Lane CL, et al. Adherence of neutrophils to canine cardiac myocytes in vitro is dependent on intercellular adhesion molecule-1. J Clin Invest 1991;88:1216-23.

7. Flavin $T$, Ivens $K$, Rothlein $R$, et al. Monoclonal antibodies against intercellular adhesion molecule 1 prolong cardiac allograft survival in cynomolgus monkeys. Transplant Proc 1991;23:533-4. 
8. Whitley D, Miyasaka M., Tamatami T, Christy M, Tilney N. Adhesion molecules in acute and chronic allograft rejection. Surg Forum 1991;42:368-71.

9. Cosimi AB, Conti D, Delmonico FL, et al. In vivo effects of monoclonal antibody to ICAM-1 (CD54) in nonhuman primates with renal allografts. J Immunol 1990;144:4604 12.

10. Pelletier RP, Miyake K, Kincade PW, Orosz CG, Ferguson RM. Inflammation-induced expression of the VCAM-1 adhesion molecule on murine cardiac endothelium. Surg Forum 1991;42:371-3.

11. Briscoe DM, Schoen FJ, Rice GE, Bevilacqua MP, Ganz P, Pober JS. Induced expression of endothelial-leukocyte adhesion molecules in human cardiac allografts. Transplantation 1991;51:537-9.

12. Christy JP, Lupinetti FM, Mardan AH, Thompson SA. Endothelial cell viability in the rat aortic wall. Ann Thorac Surg 1991;51:204-7.

13. Strickett MG, Barratt-Boyes BG, MacCulloch D. Disinfection of human heart valve allografts with antibiotics in low concentration. Pathology 1983;15:457-62.

14. Yankah AC, Dreyer W, Wottge HU, Muller-Rucholtz W, Bernhard A. Kinetics of endothelial cells of preserved aortic valve allografts used for heterotopic transplantation in inbred rat strains. In: Bodnar E, Yacoub MH, eds. Biologic and bioprosthetic valves. New York: Yorke Medical Books, 1986:73-84.

15. Tamatani T, Miyasaka M. Identification of monoclonal antibodies reactive with the rat homolog of ICAM-1, and evidence for differential involvement of ICAM-1 in the adherence of resting versus activated lymphocytes to high endothelial cells. Int Immunol 1990;2:165-71.

16. Mulligan MS, Vaporciyan AA, Miyasaka M, Tamatani T, Ward PA. TNF $\alpha$ regulates in vivo intrapulmonary expression of ICAM-1. Am J Pathol 1993;142:1739-49.

17. Elices MJ, Osborn L, Takada Y, et al. VCAM-1 on activated endothelium interacts with the leukocyte integrin VLA-4 at a site distinct from the VLA-4/fibronectin binding site. Cell 1990;60:577-84.

18. Burkly LC, Jakubowski A, Newman BM, Rosa MD, ChiRosso G, Lobb RR. Signaling by vascular cell adhesion molecule-1 (VCAM-1) through VLA-4 promotes CD3dependent T cell proliferation. Eur J Immunol 1991;21: 2871-5.

19. Hession C, Tizard R, Vassallo C, et al. Cloning of an alternate form of vascular cell adhesion molecule-1 (VCAM1). J Biol Chem 1991;266:6682-5.

20. Lupinetti FM, Tsai TT, Kneebone JM, Bove EL. The effect of cryopreservation on the presence of endothelial cells on human valve allografts. J THORAC CARDIOvaSC SURG 1993;106:912-7.

21. Fabiani B, Astarcioglu I, Guggenheim J, Tricottet V, Bismuth $\mathrm{H}$, Reynes M. Expression of major histocompatibility complex (MHC) antigens on vascular endothelium of spontaneously tolerated liver allografts. Transplant Proc 1989;21:407-8.

22. Faull RJ, Starr RJ, Russ GR. Vascular endothelial cell expression of adhesion molecules and HLA antigens in renal allografts. Transplant Proc 1989;21:316-7.

23. Issekutz TB. Inhibition of in vivo lymphocyte migration to inflammation and homing to lymphoid tissues by the TA-2 monoclonal antibody: a likely role for VLA-4 in vivo. $\mathbf{J}$ Immunol 1991;147:4178-84.

24. Carlos TM, Harlan JM. Membrane proteins involved in phagocyte adherence to endothelium. Immunol Rev 1990;114:5-28.

25. Barton RW, Rothlein R, Ksiazek J, Kennedy C. The effect of anti-intercellular adhesion molecule-1 on phorbol-esterinduced rabbit lung inflammation. J Immunol 1989; 143:1278-82.

26. Yankah AC, Wottge HU, Muller-Ruchholtz W. Prognostic importance of viability and a study of a second set allograft valve: an experimental study. J Cardiac Surg 1988;3:263-70.

27. Lupinetti FM, Cobb S, Kioschos HC, Thompson SA, Walters KS, Moore KC. Effect of immunologic differences on rat aortic valve allograft calcification. J Cardiac Surg 1992;7:65-70.

28. Gonzalez-Lavin L, Bianchi J, Graf D, Amini S, Gordon CI. Degenerative changes in fresh aortic root homografts in a canine model: evidence of an immunologic influence. Transplant Proc 1988;20(Suppl 1):815-9. 